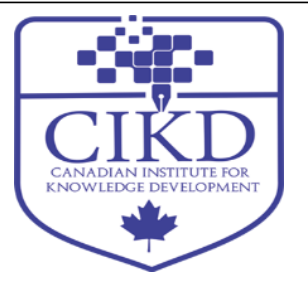

\title{
Two Cases of One-stage Procedure of TAVI and CERAB
}

\author{
Antonio Rizza*, Cataldo Palmieri, Francesco Negro, Angelo Monteleone, \\ Giuseppe Raffaele Trianni, Marcello Ravani, Sergio Berti \\ Cardiology Unit, Fondazione Toscana Gabriele Monasterio, Massa Italy
}

\begin{tabular}{|c|c|}
\hline & AbStract \\
\hline $\begin{array}{l}\text { Keywords: } \\
\text { Aortic valve stenosis, Aortic } \\
\text { bifurcation disease, TAVI, } \\
\text { CERAB }\end{array}$ & $\begin{array}{l}\text { Aortic valve stenosis (AS) and abdominal aorto-iliac stenosis typically affect elderly patients, } \\
\text { and both are associated with the adverse outcome if not adequately managed. Surgical } \\
\text { intervention is the first treatment in younger patients without significant comorbidities. } \\
\text { Notably, these conditions can be present simultaneously. The choice of the optimal treatment }\end{array}$ \\
\hline $\begin{array}{l}\text { Received } \\
\text { O7 February } 2021 \\
\text { Received in revised form } \\
22 \text { February } 2021\end{array}$ & $\begin{array}{l}\text { simultaneous treatment by percutaneous intervention, especially in high surgical-risk patients, } \\
\text { appeared to be attractive. We report a series of } 2 \text { cases with severe AS and aorto-iliac stenosis } \\
\text { referred to "Ospedale del Cuore" of Massa (Italy) who could not undergo surgical intervention. } \\
\text { They were treated with transcatheter aortic valve implantation (TAVI) and covered } \\
\text { endovascular reconstruction of aortic bifurcation (CERAB) within the same procedure. Our }\end{array}$ \\
\hline $\begin{array}{l}\text { Accepted } \\
05 \text { March } 2021\end{array}$ & $\begin{array}{l}\text { case series showed that one-stage intervention is an effective, safe, and feasible treatment of } \\
\text { these two pathologies. In the future, this approach can represent the "gold-standard" for elderly }\end{array}$ \\
\hline
\end{tabular}

rizza@ftgm.it

CCIKD Publishing

The degeneration of aortic stenosis (AS) is characterized by progressive fibro-calcific remodeling and thickening of the aortic valve leaflets that evolve over time to cause severe obstruction to cardiac outflow. While it is uncommon in patients aged under 65 years in the absence of a congenital abnormality, severe AS is the second most common valvular lesion in high-income countries. Studies suggested the incidence to be $3.4 \%$ in patients older than 75 years [1]. This pathology can be managed with transcatheter aortic valve implantation (TAVI), especially in the elderly or patients with comorbidities or surgical aortic valve replacement (SAVR).

The aortic bifurcation is an important anatomical location of atherosclerosis. Stenosis in this area could lead to disabling symptoms of peripheral arterial occlusive disease and serious 
consequences on quality of life and life-expectancy. Bilateral iliac artery obstructions involving the aortic bifurcation or high-grade stenosis of the abdominal aorta are not usually considered an indication for percutaneous treatment. However, the covered endovascular reconstruction of the aortic bifurcation (CERAB) technique was introduced in 2009 in order to provide an anatomically and physiologically optimal endovascular reconstruction of the aortic bifurcation, and the results observed during follow-up are encouraging [2].

\section{Case Presentation}

\section{Case 1}

A 73-year-old male patient was affected by hypertension, type 2 diabetes mellitus, chronic kidney disease, and obesity; in 2004, he was diagnosed with angina pectoris. Therefore a double coronary artery bypass graft (left internal mammary artery to left anterior descending coronary artery and venous graft on ottuse marginale branch) was performed. In February 2020, he had an acute coronary syndrome treated with angioplasty and drug-eluting stent implantation in the right coronary artery; during this hospital stay, he was diagnosed with moderate aortic valve stenosis (mean pressure gradient $35 \mathrm{mmHg}$ ) (Figure 1). His medical history classified the patient as a "vascular patient".

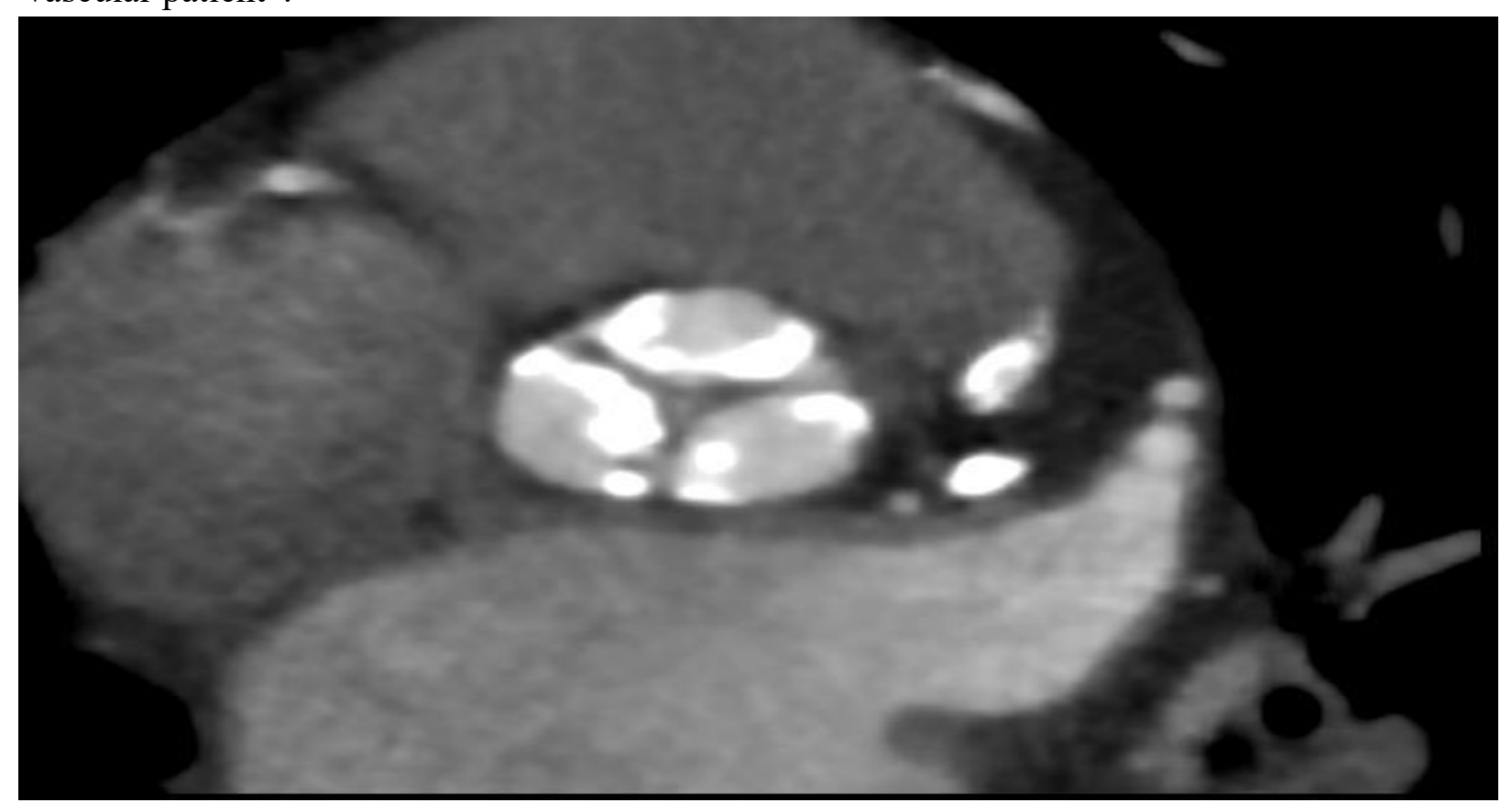

Figure 1. CT scan of aortic valve stenosis.

In December 2020, the patient had a syncope associated with an increase of serum creatinine level (up to $2.4 \mathrm{mg} / \mathrm{dl}$ ). An invasive coronary angiography was performed, showing the patency of coronary bypass and recent stent. An echocardiogram showed aortic valve disease progression from moderate to severe (mean pressure gradient: $41 \mathrm{mmHg}$ ), a normal ejection fraction $(60 \%)$ with inferior left ventricular wall hypokinesia. According to his comorbidities and the history of cardiac surgery, TAVI was preferred to SAVR. The pre-operative CT angiography showed important stenosis $(70 \%)$ of the infrarenal aorta with atherosclerotic dissected plaque involving aortic bifurcation. Since even this lesion could not be treated with an open surgical intervention, we decided to perform TAVI and CERAB in the same procedure. 
The procedure was performed in mild sedation and started with TAVI: first, ultrasound-guided left and right common femoral artery accesses were obtained, and two Perclose Proglide closure system were pre-implanted in the right one; then a pacing catheter was advanced towards the right ventricle through the left femoral vein access; a $23 \mathrm{~mm}$ Sapien 3 Ultra (Edwards) was implanted as usual and then post-dilated because of the presence of a moderate paravalvular leak; the final angiography and intra-operative echocardiogram showed a correct position of the aortic valve prosthesis and a significative reduction of the transvalvular gradient. Then, we performed CERAB: first, we positioned two endovascular prostheses $(12 \times 49 \mathrm{~mm}$ and $12 \times 39 \mathrm{~mm})$ partially imbricated in the infrarenal aorta; secondly, we implanted two covered stents $(9 \times 57 \mathrm{~mm})$ in common iliac arteries, completing the reconstruction of the aortic bifurcation. The final angiography showed a resolution of the stenosis and a normal flow towards inferior limbs (Figure 2A and 2B).

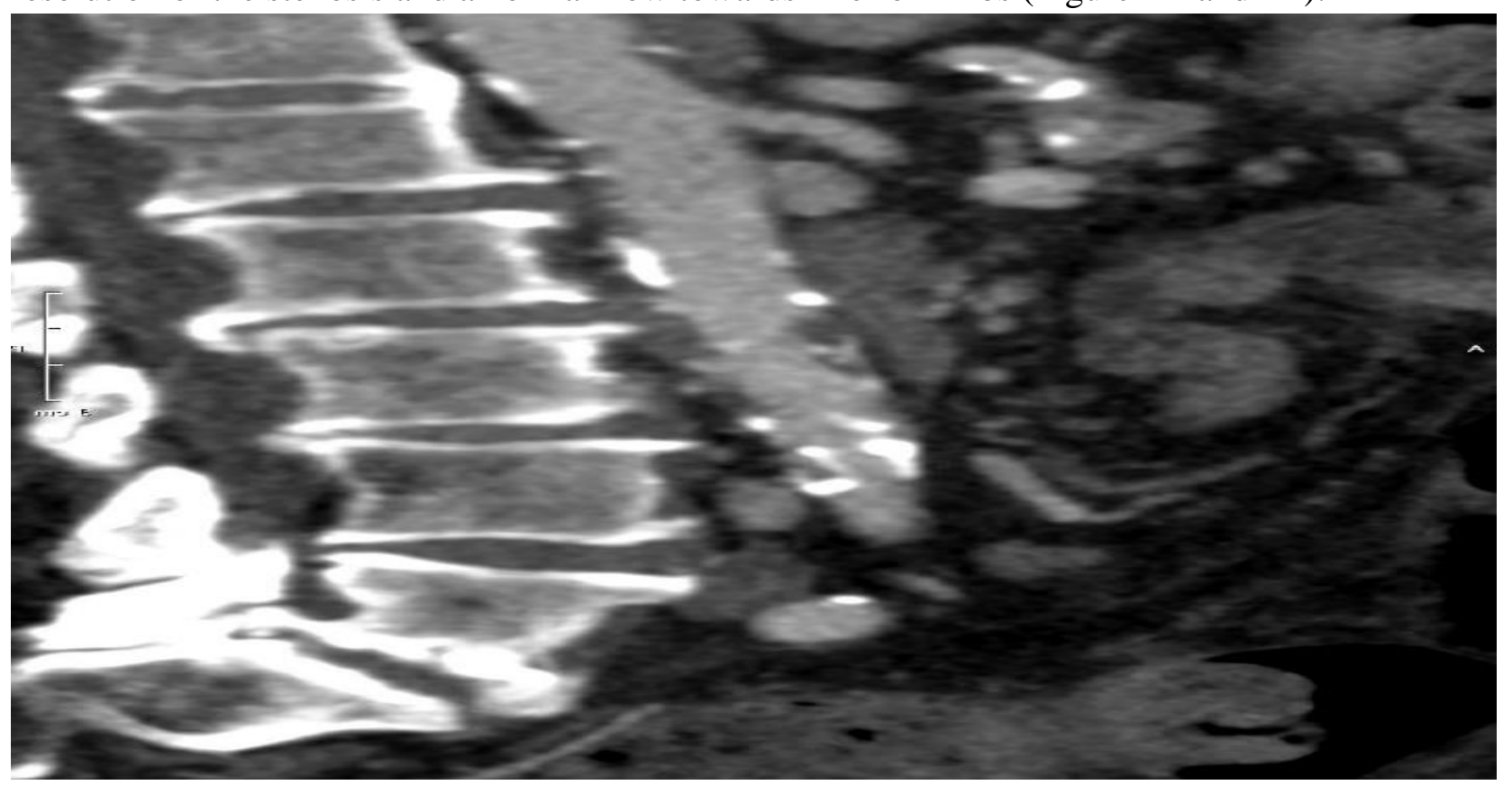

Figure 2A. Sagittal CT scan of abdominal aortic stenosis.

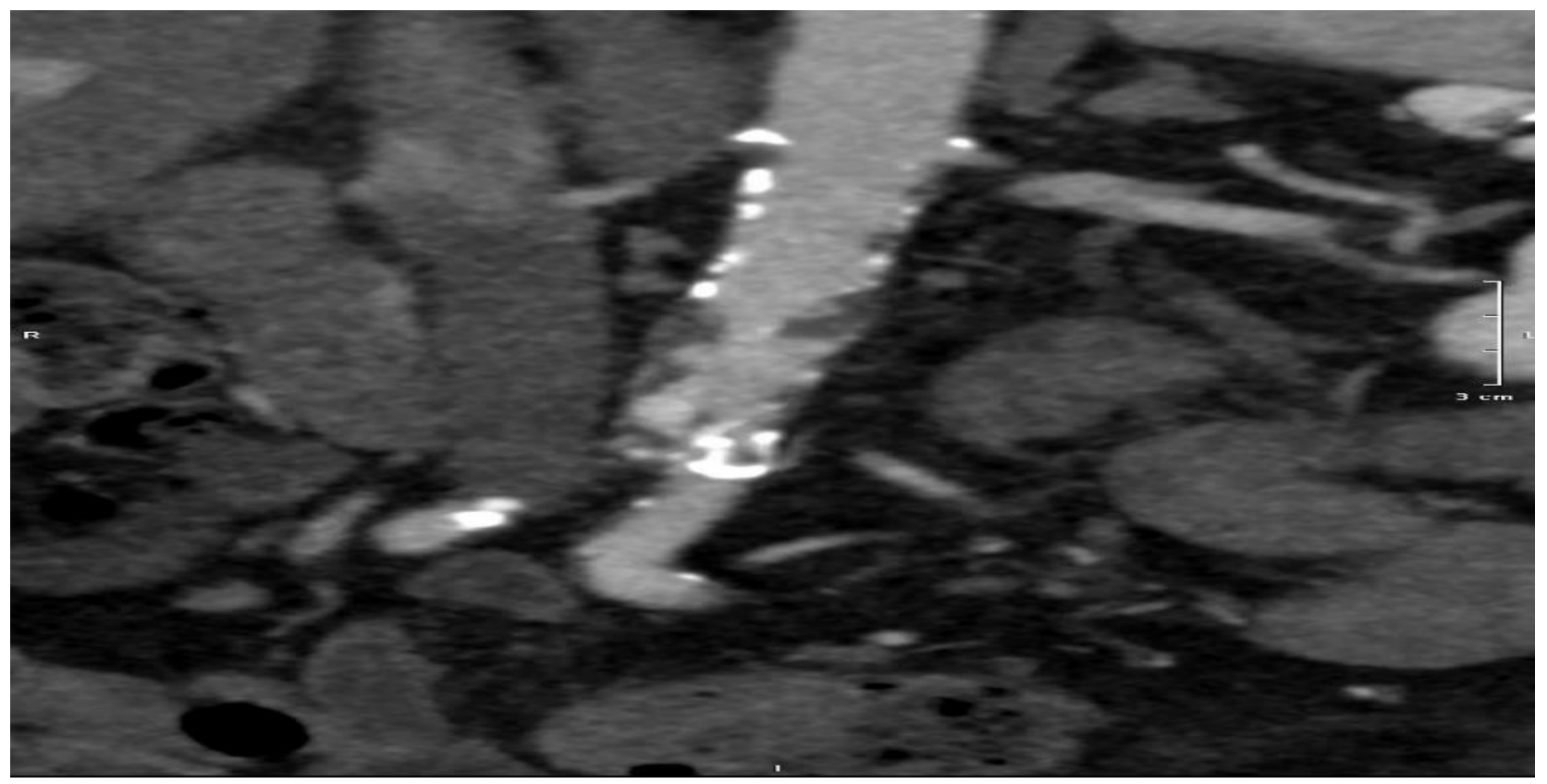

Figure 2B. Coronal CT scan of abdominal aortic stenosis. 


\section{Case 2}

A 78-year-old male patient was affected by hypertension and dyslipidemia. In 2001, he had an acute myocardial infarction treated with fibrinolysis strategy and consequent coronary artery bypass graft (right internal mammary artery to left anterior descending artery, left internal mammary artery to ottuse marginal branch, radial artery to posterior descending and venous graft to first diagonal branch). Since 2018, the patient reported effort angina without dyspnoea, but he refused to perform an invasive coronary angiography. Recently he had an ultrasound diagnosis of both severe aortic valve stenosis and regurgitation (valve area $0.8 \mathrm{cmq}$, mean pressure gradient 46 $\mathrm{mmHg}$, PHT $210 \mathrm{~ms}$ ); the ejection fraction was normal (58\%). According to the patient age and history of surgical coronary revascularization, the choice of treatment option was TAVI.

The pre-operative CT angiography showed atherosclerotic stenosis of the infrarenal aorta involving iliac arteries. Therefore, in this case, we decided to perform TAVI and CERAB in the same intervention (Figure 3A and 3B).

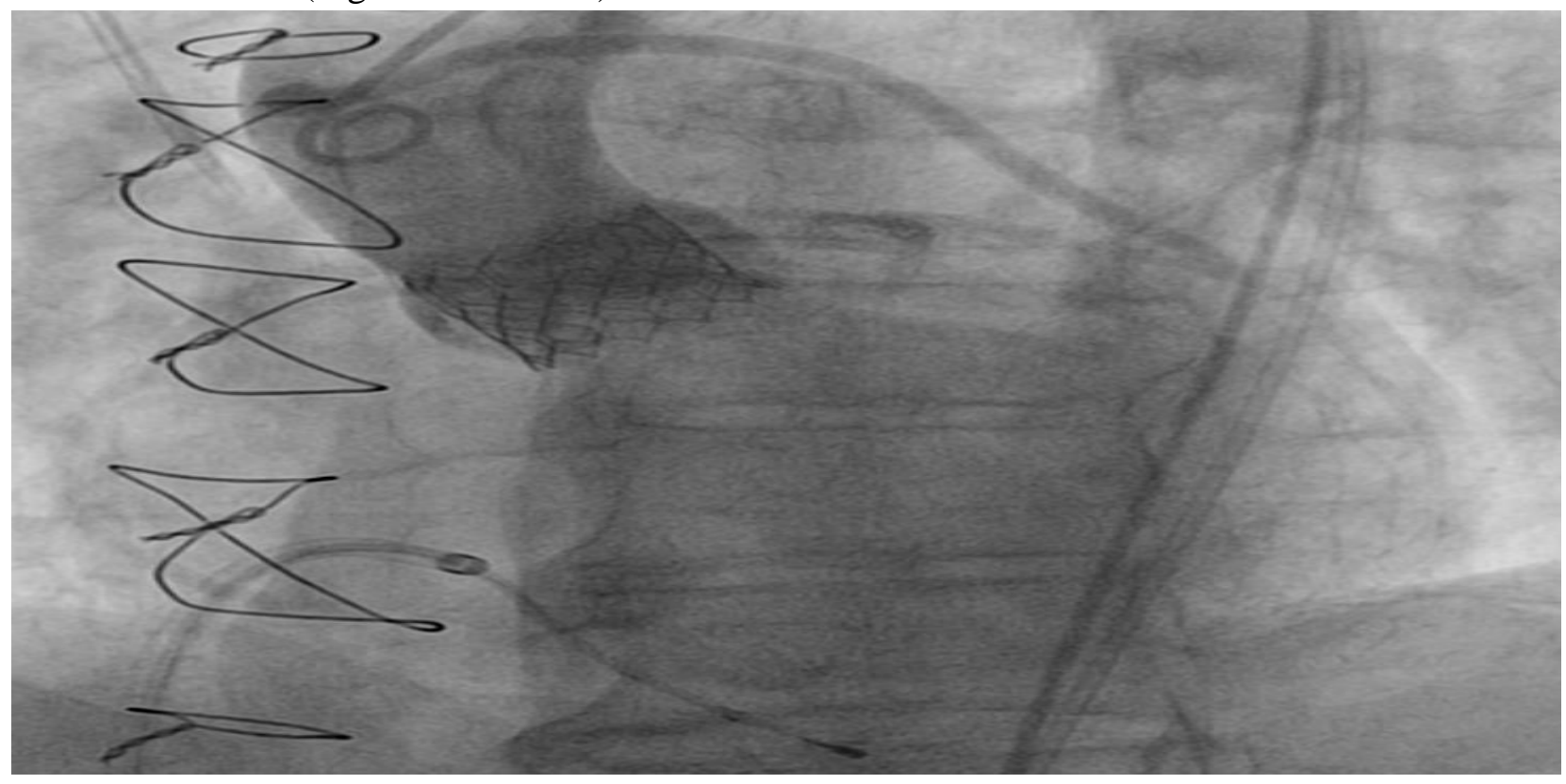

Figure 3A. TAVI final result.

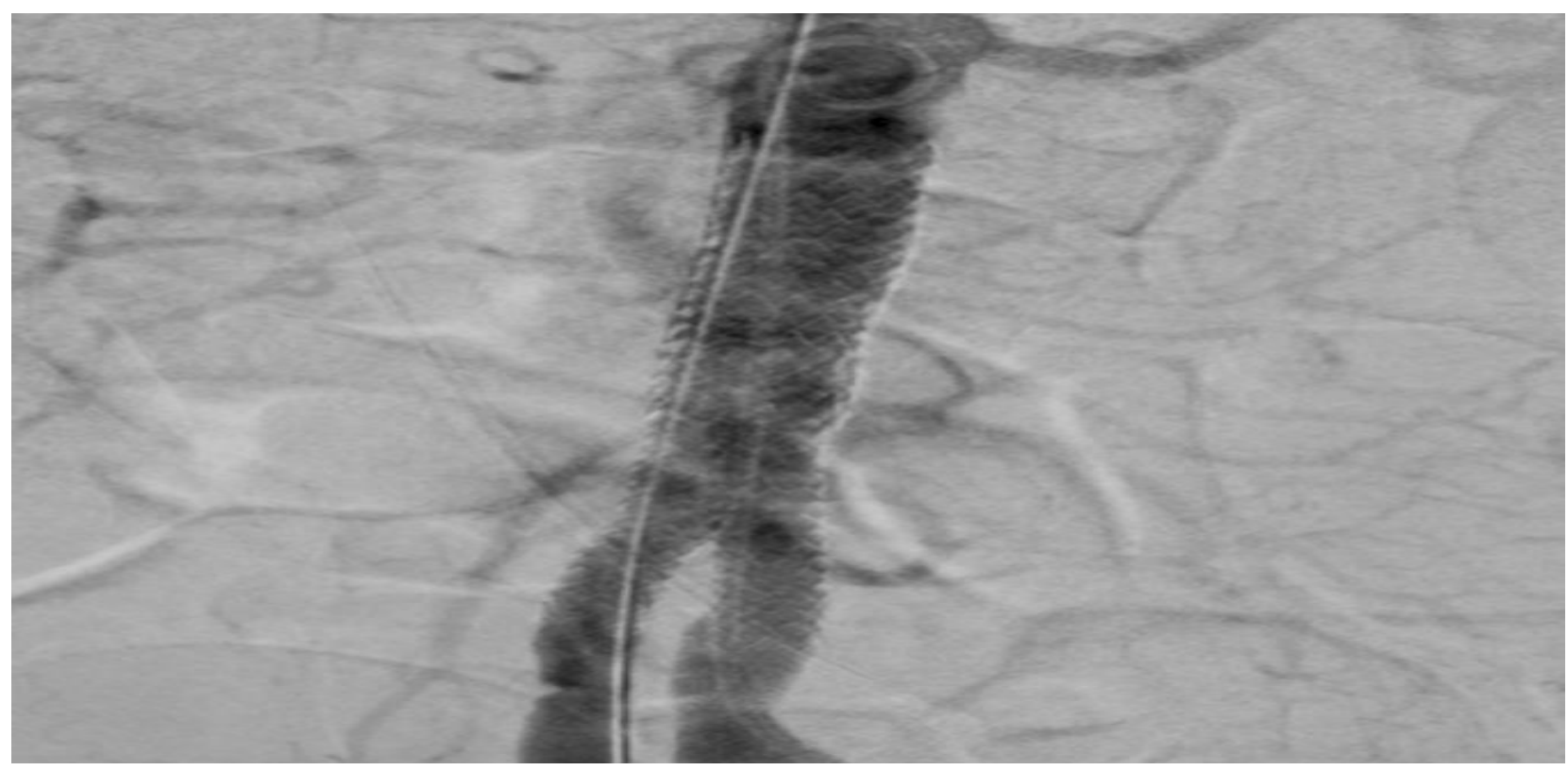

Figure 3B. CERAB final result. 
First, an invasive coronary angiography was performed to check the patency of previous coronary bypass and native coronary arteries. As this exam showed a progression of atherosclerosis in the left main stem and a circumflex coronary artery, a percutaneous angioplastystenting was executed. Then a $26 \mathrm{~mm}$ Sapien 3 Ultra (Edwards) was implanted as usual, and no leak was observed in the final angiography. Finally, we performed CERAB as follows: a BeGraft stent was positioned in the infrarenal aorta, and two other BeGraft stent $(8 \times 57 \mathrm{~mm})$ was positioned in common iliac arteries; this stage was followed by multiple balloon post-dilatations to optimize the final result.

\section{Discussion}

Aortic stenosis is the most common valve disease in the elderly. Specifically, $12.4 \%$ of subjects aged $>75$ years suffered from AS and 3.4\% from severe AS [3]. At this age, the prevalence of AAA is even higher, as it occurs in approximately $5 \%$ of men over 50 years [4].

SAVR has been the gold standard treatment for a long time but, the need for less invasive therapies developed transcatheter aortic valve implantation with an aging and an increasingly multimorbid population. The aim of surgery is to immediately restore normal aortic valve function, usually with a low incidence of paravalvular leakage (PVL), patient-prosthesis mismatch (PPM), atrioventricular blockage (AV block), and peri-operative mortality. TAVI is capable of reducing surgical trauma and avoiding cardiac arrest and cardiopulmonary bypass. TAVI is non-inferior to SAVR, and those patients who are suitable candidates for transfemoral access have an additional benefit. Randomized controlled trials demonstrated that TAVI is a therapeutic option in inoperable patients, and recently this therapeutic option has been performed successfully in moderate and lowrisk patients [5,6]. Moreover, TAVI is the treatment option in patients with bioprosthetic valve failure who face repeat SAVR with its potentially increased surgical trauma.

Aortic bifurcation stenosis prevails in a male patient with multiple cardiovascular risks. However, highly effective surgical interventions are associated with a substantial procedure-related risk for the patient. In a meta-analysis, the aggregated operative mortality rate and systemic morbidity have been attributed to $3.3 \%$ and $8.3 \%$ [7].

Three main percutaneous techniques can be an alternative to open surgery, including kissing bare metal stenting, kissing covered stenting, and the recently developed CERAB. In a vitro study, the CERAB configuration was compared with kissing self-expanding bare stents and kissing balloon-expandable covered stents [8]. These data showed that the mismatch areas and their volumes were much lower in CERAB than both kissing stent configurations, even though the stent conformation of self-expanding stents is higher, supporting the hypothesis that the CERAB configuration is the most anatomical option.

In CERAB, the time-averaged wall shear stress was two-fold lower when compared with the control model, and lesions may have a lower tendency to progress in time. The hemodynamic changes, due to the geometrical mismatches, may lead to the presence of immature mesenchymal tissue, intimal hyperplasia, and organizing thrombus not only in the space created inferior to the vertex of the opposing stents but also within the stents and the free-floating portions of the stents [9]. When comparing the three-year clinical outcome with surgical outcome, it is evident that CERAB results are very similar to open surgery.

Finding of aortic bifurcation stenotic disease and severe aortic valve stenosis is not uncommon, especially in elderly patients. According to non-cardiac surgery ESC guidelines, abdominal aorta open surgical repair is considered a high-risk procedure. When severe AS and aortic bifurcation 
stenosis coexist, this risk becomes unacceptable; therefore, aortic valve replacement should be considered before elective surgery in symptomatic patients. In patients who are high risk or contraindicated for aortic valve replacement, balloon aortic valvuloplasty or, preferably, TAVI may be a reasonable therapeutic option before surgery [10].

In the case report, we showed that it is reasonable and feasible to treat two life-threatening diseases with one less invasive procedure. Some practical details of this combined procedure need to be emphasized.

The first and obvious advantage is using the same arterial accesses for both procedures, reducing vascular complications of two different percutaneous interventions. Furthermore, this approach can reduce the number of hospitalizations and correlated risks (as nosocomial infections). In our experience, we performed TAVI first, and then we proceeded with CERAB. This strategy has been pursued because once the aortic valve prosthesis is implanted, the cardiac output improves and the risk of cerebral or peripheral hypoperfusion and endovascular prosthesis dislodgement are reduced. However, it is of great importance that the aortic bifurcation stenosis is not prohibitive to delivery aortic valve prosthesis from femoral access to the thoracic aorta. Therefore, we stress the importance of good pre-operative imaging (as CT angiography). The main disadvantage is iatrogenic renal impairment because of the use of a larger contrast medium quantity.

\section{Conclusion}

With the aging of the population, the number of patients with multiple cardiovascular diseases requiring complex interventions is likely to increase. TAVI and CERAB are two percutaneous interventional techniques to treat aortic valve stenosis and aorto-iliac bifurcation stenotic disease ("double aortic stenosis") in considerable expansion all over the world

For the first time in the literature, we report two cases of TAVI and CERAB performed in a single procedure, showing a safe and feasible intervention in patients at high surgical risk. In the next future, this approach could reduce multiple hospitalizations and periprocedural complications.

\section{Conflict of Interest}

All the authors declare that no grant or other form of financial support was received regarding this manuscript. All the authors declare no conflict of interest regarding this work.

\section{References}

[1] Osnabrugge RL, Mylotte D, Head SJ, Van Mieghem NM, Nkomo VT, LeReun CM, et al. Aortic stenosis in the elderly: disease prevalence and number of candidates for transcatheter aortic valve replacement: a meta-analysis and modeling study. J Am Coll Cardiol. 2013;62(11):1002-12.

[2] Taeymans K, Groot Jebbink E, Holewijn S, Martens JM, Versluis M, Goverde PCJM, Reijnen MMPJ. Three-year outcome of the covered endovascular reconstruction of the aortic bifurcation technique for aortoiliac occlusive disease. J Vasc Surg. $2018 ; 67(5): 1438-1447$.

[3] Thoenes M, Bramlage P, Zamorano P, Messika-Zeitoun D, Wendt D, Kasel M, et al. Patient screening for early detection of aortic stenosis (AS)-review of current practice and future perspectives. J Thorac Dis. 2018;10(9):5584-5594.

[4] Davis M, Taylor PR. Endovascular infrarenal abdominal aortic aneurysm repair. Heart. 2008;94(2):222-8. 
[5] Mack MJ, Leon MB, Thourani VH, Makkar R, Kodali SK, Russo M, et al. PARTNER 3 Investigators. Transcatheter Aortic-Valve Replacement with a Balloon-Expandable Valve in Low-Risk Patients. N Engl J Med. 2019;380(18):16951705.

[6] Leon MB, Smith CR, Mack MJ, Makkar RR, Svensson LG, Kodali SK, et al. PARTNER 2 Investigators. Transcatheter or Surgical Aortic-Valve Replacement in Intermediate-Risk Patients. N Engl J Med. 2016;374(17):1609-20.

[7] de Vries SO, Hunink MG. Results of aortic bifurcation grafts for aortoiliac occlusive disease: a meta-analysis. J Vasc Surg. $1997 ; 26(4): 558-69$.

[8] Groot Jebbink E, Grimme FA, Goverde PC, van Oostayen JA, Slump CH, Reijnen MM. Geometrical consequences of kissing stents and the Covered Endovascular Reconstruction of the Aortic Bifurcation configuration in an in vitro model for endovascular reconstruction of aortic bifurcation. J Vasc Surg. 2015;61(5):1306-11.

[9] Saker MB, Oppat WF, Kent SA, Ryu RK, Chrisman HB, Nemcek AA, et al. Early failure of aortoiliac kissing stents: histopathologic correlation. J Vasc Interv Radiol. 2000 Mar;11(3):333-6.

[10] Kristensen SD, Knuuti J, Saraste A, Anker S, Bøtker HE, Hert SD, et al. 2014 ESC/ESA Guidelines on non-cardiac surgery: cardiovascular assessment and management: The Joint Task Force on non-cardiac surgery: cardiovascular assessment and management of the European Society of Cardiology (ESC) and the European Society of Anaesthesiology (ESA). Eur Heart J. 2014;35(35):2383-431. 